\title{
Relationship Between Hemorheology Assessed Using Microchannel Array Flow Analyzer and Kidney Function in Hypertensive Patients
}

\author{
Takashi Hitsumoto
}

\begin{abstract}
Background: Kidney function is known to be closely associated with the pathogenesis of hypertension. In contrast, hemorheology assessed using microchannel array flow analyzer (MC-FAN) has demonstrated the significance of cardiovascular risk factors in recent clinical studies. The present cross-sectional study aimed to clarify the relationship between hemorheology assessed by MC-FAN and kidney function in hypertensive patients from the perspective of primary prevention of cardiovascular events.
\end{abstract}

Methods: In total, 453 outpatients undergoing treatment for hypertension (176 men and 277 women; mean age \pm standard deviation: $65 \pm 13$ years) with no history of cardiovascular disease were enrolled. Whole blood passage time (WBPT) was measured using MC-FAN as a marker of hemorheology, and the relationships with various clinical parameters including kidney function were examined.

Results: A significant correlation was observed between WBPT and the parameters of kidney function such as estimated glomerular filtration rate $(\mathrm{r}=-0.14, \mathrm{P}<0.01)$, urinary albumin excretion $(\mathrm{r}=0.40, \mathrm{P}$ $<0.001)$, and renal resistive index $(\mathrm{r}=0.44, \mathrm{P}<0.001)$. Furthermore, multivariate analysis demonstrated urinary albumin excretion, renal resistive index, skin autofluorescence, derivatives of reactive oxygen metabolites, and hematocrit as independent variables for WBPT as a subordinate factor.

Conclusions: The results of the present study indicate that hemorheology assessed by the MC-FAN is significantly associated with markers of kidney function, such as albuminuria and increased renovascular resistance, in hypertensive patients.

Keywords: Hemorheology; Microchannel method; Albuminuria; Renal resistive index; Hypertension

Manuscript submitted July 4, 2017, accepted July 14, 2017

Hitsumoto Medical Clinic, 2-7-7, Takezakicyou, Shimonoseki City, Yamaguchi 750-0025, Japan. Email: thitsu@jcom.home.ne.jp

doi: https://doi.org/10.14740/cr572w

\section{Introduction}

Kidney function is known to be closely associated with the pathogenesis of hypertension. Clinically, kidney function can be evaluated using biomarkers such as estimated glomerular filtration rate (eGFR) and urinary albumin concentration. These biomarkers are also well established as prognosis factors for cardiovascular events [1]. In addition, renal resistive index (RRI), which is estimated by ultrasonography, has been reported as a novel marker of kidney function and a predictor of cardiovascular events [2].

The impairment of hemorheology is an important factor in the incidence of cardiovascular events as well as atherosclerosis $[3,4]$. Recently, a commercial device called the microchannel array flow analyzer (MC-FAN), which evaluates hemorheology using microscopic images, has been introduced to clinical settings [5]. The use of MC-FAN is simple, and it is superior to other methods in terms of the accuracy of channel dimensions and high reproducibility. Furthermore, clinical studies have reported significant relationship between an increase in the whole blood passage time (WBPT), which can be measured using MC-FAN, and cardiovascular risk factors or coronary artery disease [6-8].

To the best of our knowledge, no reports exist on the relationship between WBPT and kidney function in hypertensive patients. Therefore, the present study examined the relationship between WBPT and kidney function parameters, such as eGFR, urinary albumin excretion, and RRI in hypertensive patients from the perspective of primary prevention of cardiovascular events.

\section{Methods}

\section{Patients}

The present cross-sectional study was conducted at the Hitsumoto Medical Clinic in Shimonoseki City from December 2014 to November 2016. The study population comprised 453 outpatients undergoing treatment for hypertension whose blood rheology was examined using MC-FAN. No patient had a history of cardiovascular events, such as coronary artery disease, ischemic stroke, or peripheral artery disease. Study par- 
ticipants comprised 176 men and 277 women with a mean age \pm standard deviation (SD) of $65 \pm 13$ years. All participants provided informed consent, and the study protocol conformed to the ethical guidelines of the Declaration of Helsinki. The present study was approved by the Local Ethics Committee of the Hitsumoto Medical Clinic.

\section{Evaluation of hemorheology using MC-FAN}

The evaluation of hemorheology was performed by measuring WBPT using an MC-FAN HR300 rheometer (MC Healthcare Inc., Tokyo, Japan), as previously reported [5, 9]. The microchannel passage time for $100 \mu \mathrm{L}$ of physiological saline as a control was initially measured, followed by that for $100 \mu \mathrm{L}$ of heparinized blood samples obtained from the study participants. WBPT for participants was corrected for the passage time of physiological saline. The microchannel formation was 7- $\mu \mathrm{m}$ wide, $30-\mu \mathrm{m}$ long, and 4.5- $\mu \mathrm{m}$ deep. WBPT measurements were performed within $60 \mathrm{~min}$ of blood sampling. Interand intra-assay coefficients of variation for WBPT were $8 \%$ and $5 \%$, respectively.

\section{Evaluation of kidney function}

The eGFR was calculated using the adjusted Modification of Diet in Renal Disease Study equation, which was proposed by the working group of the Japanese Chronic Kidney Disease Initiative [10]. Urinary albumin excretion was evaluated using a commercial kit (Siemens/Bayer DCA 2000+ Analyzer, Siemens Healthineers, Tokyo, Japan). Ultrasonographic examination of renal arteries was performed using a $3.0-\mathrm{MHz}$ convex probe (HI VISION Avius, Hitachi Medical Corporation, Tokyo, Japan). RRI was measured as previously reported [11]. Measurement was performed in a supine position during suspended respiration at the end of inspiration. Using pulsed wave Doppler, blood flow velocities were measured from segmental arteries located in the upper, middle, and lower thirds of the kidney for RRI analysis. The RRI was automatically calculated using the following equation: (peak systolic velocity - minimum diastolic velocity)/peak systolic velocity. The three values were averaged to obtain the mean RRI for each kidney. The average value between the right and left kidney was the RRI used for analysis.

\section{Evaluation of cardiovascular risk factors}

The degree of obesity was estimated using body mass index, which was calculated as weight in kilograms divided by height in meters squared. Current smoking was defined as smoking at least one cigarette per day during the previous 28 days. Right brachial blood pressure was measured twice using a mercury sphygmomanometer with participants in the sitting position. An average of two readings was used to determine systolic and diastolic blood pressures. Skin autofluorescence, which reflects the accumulation of advanced glycation end products (AGEs), was measured on the volar side of the forearm us-
Table 1. Baseline Clinical Characteristics

\begin{tabular}{|c|c|}
\hline n (male/female) & $453(176 / 277)$ \\
\hline Age (years) & $65 \pm 13$ \\
\hline Body mass index & $23.0 \pm 3.7$ \\
\hline Current smoker, n (\%) & $105(23)$ \\
\hline Systolic blood pressure (mm Hg) & $156.4 \pm 8.1$ \\
\hline Diastolic blood pressure (mm Hg) & $90.4 \pm 9.2$ \\
\hline Pulse rate (/min) & $66 \pm 10$ \\
\hline White blood cell $(/ \mu \mathrm{L})$ & $6,580 \pm 1,250$ \\
\hline Red blood cell $\left(10^{4} / \mu \mathrm{L}\right)$ & $424 \pm 39$ \\
\hline Hematocrit (\%) & $38.1 \pm 3.9$ \\
\hline Platelet $\left(10^{4} / \mu \mathrm{L}\right)$ & $21.6 \pm 6.2$ \\
\hline Fasting blood glucose (mg/dL) & $113 \pm 24$ \\
\hline Immunoreactive insulin $(\mu \mathrm{g} / \mathrm{mL})$ & $7.3 \pm 4.4$ \\
\hline HOMA-IR & $2.1 \pm 1.4$ \\
\hline Skin autofluorescence (AU) & $2.5 \pm 0.6$ \\
\hline Total cholesterol (mg/dL) & $212 \pm 41$ \\
\hline LDL-cholesterol (mg/dL) & $135 \pm 36$ \\
\hline Triglyceride (mg/dL) & $128 \pm 64$ \\
\hline HDL-cholesterol (mg/dL) & $52 \pm 14$ \\
\hline d-ROMs test (U Carr) & $331 \pm 96$ \\
\hline eGFR $\left(\mathrm{mL} / \mathrm{min} / 1.73 \mathrm{~m}^{2}\right)$ & $66.0 \pm 21.2$ \\
\hline Log-urinary albumin (mg/g Cr) & $1.69 \pm 0.63$ \\
\hline RRI & $0.7 \pm 0.1$ \\
\hline WBPT (s) & $61.2 \pm 16.0$ \\
\hline \multicolumn{2}{|l|}{ Medication } \\
\hline RAS inhibitor, n (\%) & $245(54)$ \\
\hline Calcium channel blocker, n (\%) & $308(68)$ \\
\hline$\beta$-blocker, n (\%) & $36(8)$ \\
\hline Statin, n (\%) & $236(52)$ \\
\hline Eicosapentaenoic acid, n (\%) & $18(4)$ \\
\hline
\end{tabular}

Continuous values are mean \pm SD. HOMA-IR: homeostasis assessment insulin resistance; LDL: low-density lipoprotein; HDL: high-density lipoprotein; d-ROMs: derivatives of reactive oxygen metabolites; eGFR: estimated glomerular filtration rate; RR: renal resistive index; WBPT: whole blood passage time; RAS: renin-angiotensin system.

ing a commercial instrument (AGE ReaderTM; DiagnOptics, Groningen, The Netherlands), as previously described [12]. Blood cell counts, plasma glucose concentration, plasma insulin concentration, serum lipid concentration, and derivatives of reactive oxygen metabolites (d-ROMs) were measured using d-ROMs test. Blood samples were collected from antecubital veins in the morning after $12 \mathrm{~h}$ of fasting. Glucose and insulin concentrations were measured using the glucose oxidase method and an enzyme immunoassay, respectively. To estimate insulin resistance, the homeostasis model assessment (HOMA-IR) was calculated as follows [13]: HOMA-IR $=($ fasting glucose concentration $(\mathrm{mg} / \mathrm{dL}) \times$ fasting insulin 

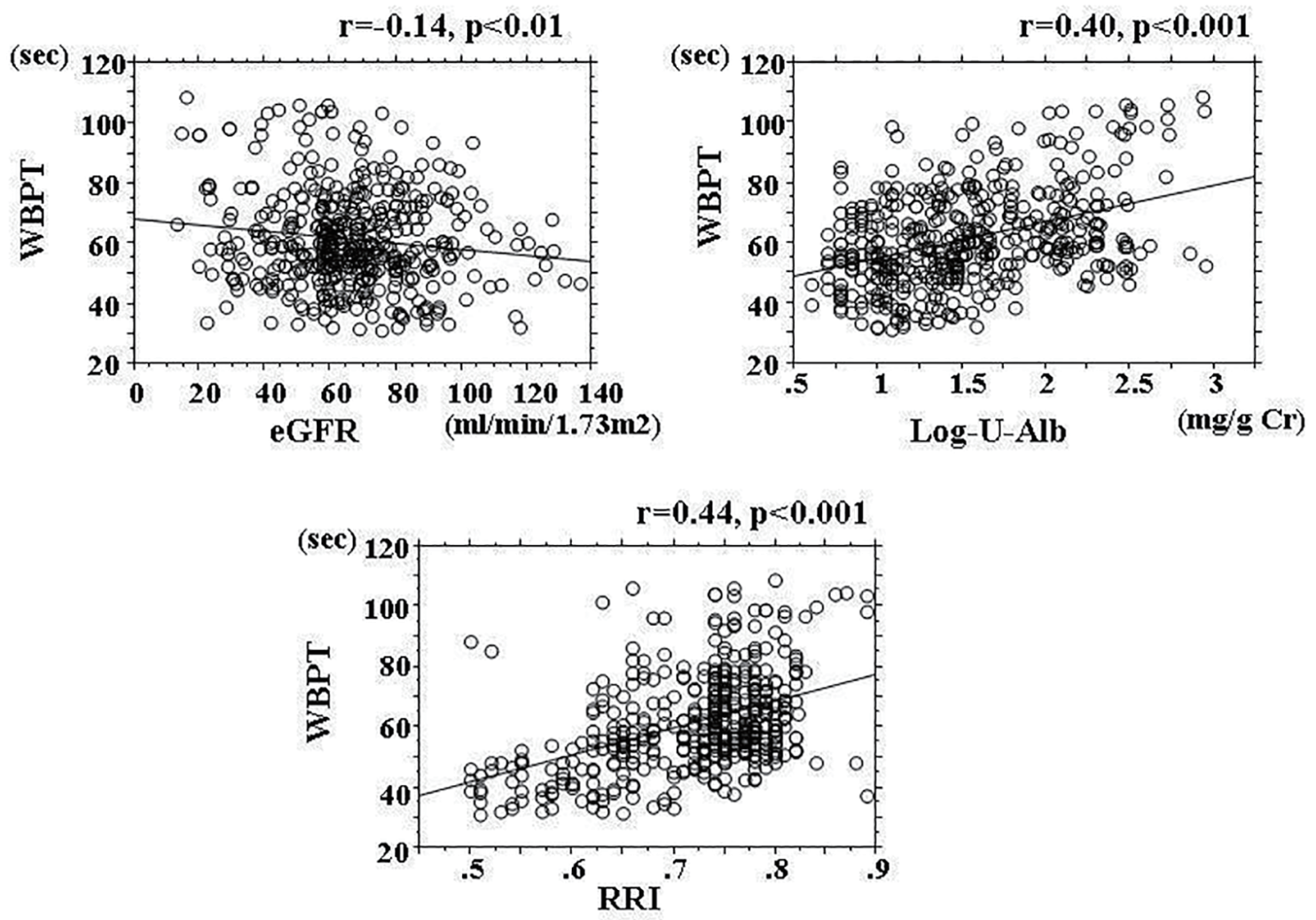

Figure 1. Correlation between WBPT and markers of kidney function. WBPT: whole blood passage time; eGFR: estimated glomerular filtration rate; RRI: renal resistive index.

concentration $(\mu \mathrm{g} / \mathrm{mL})) / 405$. Total cholesterol and triglyceride concentrations were measured using standard enzymatic methods. High- and low-density lipoprotein cholesterol concentrations were measured using selective inhibition and the Friedewald formula, respectively [14]. Participants with a serum triglyceride concentration of $\geq 400 \mathrm{mg} / \mathrm{dL}$ were excluded as this method is accurate only below this concentration. The d-ROMs test, which measures hydroperoxide levels, was used to measure oxidative stress in vivo using a commercial device (Diacron, Grosseto, Italy) [15].

\section{Statistical analysis}

A commercially available statistical software program (StatView-J 5.0; Hulinks Inc., Tokyo, Japan) was used for all statistical analyses. Continuous variables were expressed as means \pm SD. Simple regression analysis was performed using Spearman rank correlation. Multivariate analysis was performed using multiple regression or multiple logistic regression analysis. P-values $<0.05$ were considered statistically significant.

\section{Results}

Baseline clinical characteristics are shown in Table 1. The mean and median values of WBPT were 61.2 and $63.1 \mathrm{~s}$ (SD:
$16.0 \mathrm{~s}$; range: 31.1 - $108.6 \mathrm{~s}$ ), respectively. The distribution of WBPT was close to normal. The correlation between WBPT and markers of kidney function is shown in Figure 1. WBPT had a statistically significant negative correlation with eGFR and a statistically significant positive correlation with urinary albumin excretion, RRI. Correlations between WBPT and various clinical parameters are shown in Table 2. No relationship was observed between blood pressure levels and WBPT. Body mass index, current smoking status, red blood cell count, hematocrit, fasting blood glucose levels, skin autofluorescence, serum triglyceride concentration, and d-ROMs test values were significantly correlated with WBPT.

Multiple regression analysis of WBPT as a subordinate factor was performed with explanatory variables that were significant on univariate analysis. Urinary albumin excretion, RRI, skin autofluorescence, d-ROMs test values, and hematocrit were selected as independent variables for WBPT (Table 3 ). To clarify the simple threshold of WBPT for detecting albuminuria or high RRI, participants were divided into three groups based on WBPT, and multiple logistic regression analysis was performed (Fig. 2). Albuminuria was defined as a urinary albumin excretion of $\geq 30 \mathrm{mg} / \mathrm{g} \mathrm{Cr}$. Patients with high WBPT ( $\geq 70.0 \mathrm{~s})$ and median WBPT (50.0 - $70.0 \mathrm{~s})$ had significantly higher risk (odds ratio (OR): $3.1 ; 95 \%$ confidence interval (CI): 1.6 - 6.0; $\mathrm{P}<0.001$ and OR: $2.3 ; 95 \% \mathrm{CI}: 1.3$ - 4.2; P $<0.01$, respectively) of being detected with albuminuria than those with low WBPT $(\leq 50.0 \mathrm{~s})$. On the other hand, high RRI 
Table 2. Relationship Between WBPT and Various Clinical Parameters

\begin{tabular}{lll}
\hline & r & P value \\
\hline Sex $($ female $=0$, male $=1)$ & 0.07 & 0.138 \\
Age & 0.06 & 0.189 \\
Body mass index & 0.14 & $<0.01$ \\
Current smoker $($ no $=0$, yes $=1)$ & 0.13 & $<0.01$ \\
Systolic blood pressure & 0.03 & 0.469 \\
Diastolic blood pressure & 0.05 & 0.245 \\
Pulse rate & 0.06 & 0.229 \\
White blood cell & 0.06 & 0.238 \\
Red blood cell & 0.10 & $<0.05$ \\
Hematocrit & 0.13 & $<0.01$ \\
Platelet & 0.08 & 0.065 \\
Fasting blood glucose & 0.11 & $<0.05$ \\
Immunoreactive insulin & 0.05 & 0.263 \\
HOMA-IR & 0.06 & 0.187 \\
Skin autofluorescence & 0.41 & $<0.001$ \\
Total cholesterol & 0.02 & 0.627 \\
LDL-cholesterol & 0.02 & 0.604 \\
Triglyceride & 0.10 & $<0.05$ \\
HDL-cholesterol & 0.08 & 0.601 \\
d-ROMs test & 0.40 & $<0.001$ \\
RAS inhibitor $($ no $=0$, yes $=1)$ & -0.08 & 0.072 \\
CCB $($ no $=0$, yes $=1)$ & 0.05 & 0.251 \\
B-blocker $($ no $=0$, yes $=1)$ & 0.07 & 0.153 \\
Statin (no =0, yes $=1$ ) & -0.09 & 0.051 \\
Eicosapentaenoic acid $($ no $=0$, yes $=1)$ & -0.08 & 0.062 \\
\hline & & \\
\hline
\end{tabular}

$r$ expressed correlation coefficient. HOMA-IR: homeostasis assessment insulin resistance; LDL: low-density lipoprotein; HDL: high-density lipoprotein; d-ROMs: derivatives of reactive oxygen metabolites; eGFR: estimated glomerular filtration rate; RRI: renal resistive index; WBPT: whole blood passage time; RAS: renin-angiotensin system.

was defined as an RRI $\geq 0.7$. Patients with high WBPT and median WBPT had significantly higher risk (OR: 5.4; 95\% CI: 1.8 - 16.8; $\mathrm{P}<0.01$ and OR: 3.2; 95\% CI: $1.1-9.7$; $\mathrm{P}<0.05$, respectively) of having a high RRI than those with low WBPT.

\section{Discussion}

This study aimed to clarify the relationship between hemorheology assessed by the microchannel method and kidney function in hypertensive patients in terms of the primary prevention of cardiovascular events. The results of multivariate analysis demonstrated a significant relationship between WBPT as a marker of hemorheology and urinary albumin excretion, measured as the RRI. Thus, increased WBPT was found to be closely associated with markers of progression of kidney
Table 3. Multiple Regression Analysis for WBPT

\begin{tabular}{llll}
\hline Explanatory factor & $\boldsymbol{\beta}$ & t value & P value \\
\hline Log-urinary albumin & 0.33 & 8.3 & $<0.001$ \\
RRI & 0.27 & 6.5 & $<0.001$ \\
Skin autofluorescence & 0.24 & 5.7 & $<0.001$ \\
d-ROMs test & 0.22 & 5.3 & $<0.001$ \\
Hematocrit & 0.10 & 2.2 & $<0.05$ \\
eGFR & 0.09 & 1.9 & 0.061 \\
Red blood cell & 0.08 & 1.8 & 0.078 \\
Body mass index & 0.06 & 1.6 & 0.104 \\
Current smoker & 0.06 & 1.5 & 0.121 \\
Fasting blood glucose & 0.03 & 0.8 & 0.393 \\
Triglyceride & -0.01 & -0.1 & 0.704 \\
\hline
\end{tabular}

$R^{2}=0.38, F$ value $=24.7, P<0.001, n=453$. WBPT: whole blood passage time; RRI: renal resistive index; d-ROMs: derivatives of reactive oxygen metabolites; eGFR: estimated glomerular filtration rate.

dysfunction such as albuminuria and increased renovascular resistance in hypertensive patients. A recent clinical study using MC-FAN reported that the mean WBPT values in healthy control subjects with mean blood pressure levels of 113.2/71.5 $\mathrm{mm} \mathrm{Hg}$ (systolic/diastolic) were $37.1 \mathrm{~s}$ [16]. The mean WBPT in hypertensive patients with mean blood pressure levels of 156.4/90.4 mm Hg in the present study was $61.2 \mathrm{~s}$. However, blood pressure levels had no relationship with WBPT in this study. Therefore, WBPT in hypertensive patients may be associated with other factors rather than high blood pressure levels.

The mechanisms underlying the development of albuminuria are not completely understood. However, recent basic studies using animal models have reported that impairment of preglomerular small vessels, especially the perforating arteries, plays a crucial role in the incidence of urinary albumin [17]. These vessels may be injured under exposure to high blood pressures due to their small length and diameter. On the other hand, hemorheology estimated using MC-FAN is an in vitro measurement that uses artificial blood vessels, with a vessel lumen measuring a width of $7 \mu \mathrm{m}$, a length of $30 \mu \mathrm{m}$, and a depth of $4.5 \mu \mathrm{m}$. Thus, evaluating hemorheology using MC-FAN is assumed to correspond to small vessels, including perforating arteries. Other studies have indicated that urinary albumin concentration is closely associated with systemic endothelial dysfunction [18]. In addition, Yagi et al reported a significant association between increased WBPT and endothelial dysfunction using flow-mediated dilatation of the brachial artery [19]. Therefore, the significant relationship observed between WBPT and urinary albumin excretion in this study may be partly attributable to endothelial dysfunction due to impairment of hemorheology at renal small vessels including perforating arteries.

RRI is considered to reflect renovascular resistance distal from the point of examination. Ikee et al reported the relationship between RRI and renal histopathologic data, including glomerular sclerosis, interstitial fibrosis/tubular atrophy, interstitial infiltration, and arteriosclerosis. They concluded that 

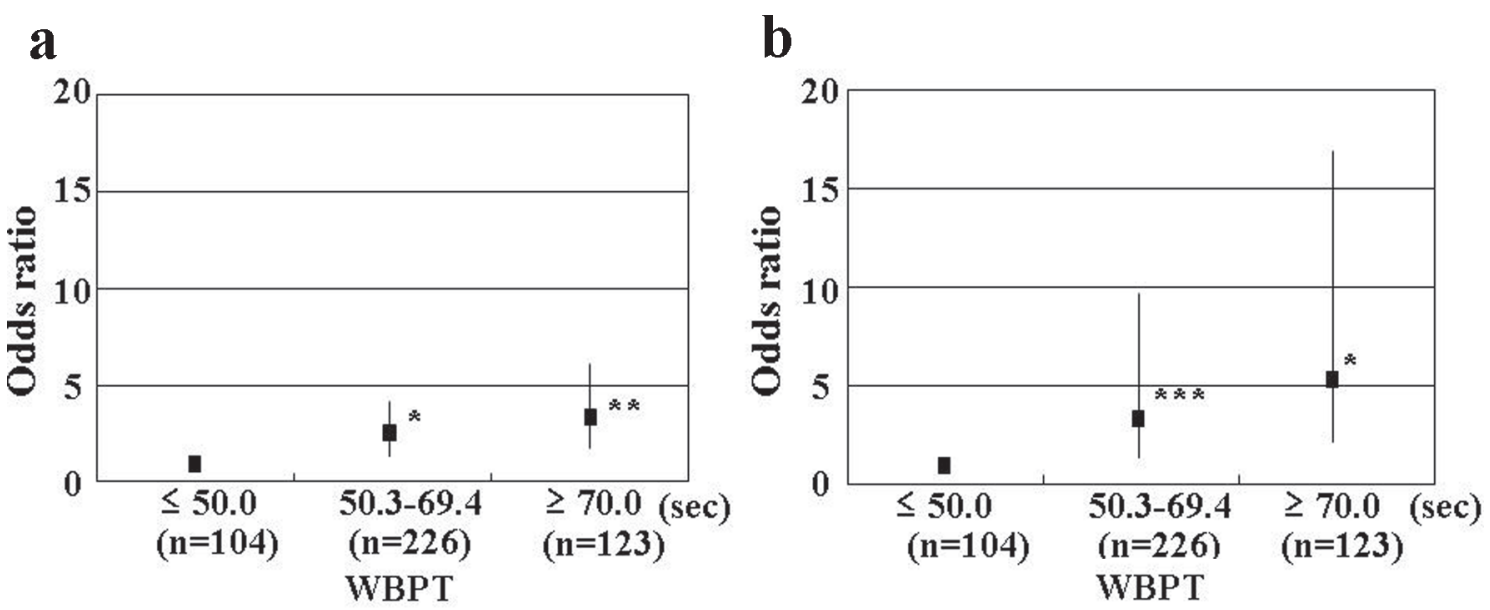

Figure 2. Results of multiple logistic regression analysis for albuminuria and high RRI. (a) Albuminuria (urinary albumin excretion as $\geq 30 \mathrm{mg} / \mathrm{g} \mathrm{Cr}$ ) as the subordinate factor. Adjustment factors were age, eGFR, skin autofluorescence, RRI, d-ROMs, and hematocrit. (b) High RRI (RRI $\geq 0.7)$ as the subordinate factor. Adjustment factors were age, eGFR, skin autofluorescence, urinary albumin excretion, d-ROMs, and hematocrit. ${ }^{*} \mathrm{P}<0.01 \mathrm{vs}$. $\leq 50.0 \mathrm{~s}$; ${ }^{* *} \mathrm{P}<0.001 \mathrm{vs}$. $\leq 50.0 \mathrm{~s}$; ${ }^{* *} \mathrm{P}<0.05 \mathrm{vs}$. $\leq 50.0 \mathrm{~s}$. RRI: renal resistive index; WBPT: whole blood passage time; eGFR: estimated glomerular filtration rate; d-ROMs: derivatives of reactive oxygen metabolites.

only arteriosclerosis was selected as an independent variable for RRI by multivariate analysis [20]. On the other hand, Linde et al reported significant relationship between blood viscosity and peripheral vascular resistance in hypertensive patients [21]. In addition, the present study indicated that WBPT is significantly associated with RRI. Thus, increased renovascular resistance may cause both impairment of blood rheology and arteriosclerosis of renal small arteries.

It is well known that AGEs have an important role in the pathogenesis of kidney disease [22, 23]. On the other hand, the level of skin pentosidine, which is a major component of AGEs on the volar side of the forearm, has been found to correlate with skin autofluorescence on skin biopsy [24]. Smallsized vessels are considered to be present in a portion of skin biopsies. Therefore, the significant relationship between skin autofluorescence and WBPT possibly reflects the accumulation of AGEs in tissues due to impairment of hemorheology in small-sized vessels. In addition, basic studies have reported that AGEs influence hemorheology by mechanisms such as leukocyte-endothelial interaction and platelet aggregation [25, 26]. Thus, the results of the present study and that of previous studies may indicate an association between AGEs and the impairment of hemorheology not only in small-sized vessels on the volar side of the forearm, but also in renal small vessels, consequently leading to the progression of kidney dysfunction in hypertensive patients.

A number of studies have reported an association between oxidative stress and the pathogenesis of hypertension [27, 28]. On the other hand, basic and clinical studies have reported that oxidative stress plays an important role in blood rheology $[7,29,30]$. In the present study, the d-ROMs test as a marker of oxidative stress in vivo also exhibited a significant correlation with the WBPT by multivariate analysis. Oxidative stress causes the impairment of hemorheology through several mechanisms, such as platelet aggregation and increased plasma viscosity $[31,32]$. The results of the present cross-sectional study also indicate that oxidative stress plays an important role in the impairment of renal microcirculation in hypertensive patients.

Validated WBPT values for predicting the progression of kidney dysfunction are required in clinical settings. In one study, to clarify the clinical usefulness of measuring WBPT to detect microalbuminuria or high RRI, as reported by previous studies, participants were divided into three groups based on simple WBPT values and multiple logistic regression analysis was performed $[1,2,11]$. The results of the present study indicate that patients with WBPT $\geq 70.0 \mathrm{~s}$ or $50.0-70.0 \mathrm{~s}$ exhibited a significantly higher risk of urinary albuminuria and high RRI than patients with WBPT $\leq 50.0 \mathrm{~s}$. Although this was a cross-sectional study, we believe that it is possible to evaluate the risk of progression of kidney dysfunction in hypertensive patients by measuring WBPT. Some clinical studies have indicated that medications, such as anti-hypertensive drugs and anti-hyperlipidemic drugs, and smoking cessation improve hemorheology; however, no statistically significant association between these factors and hemorheology was observed in this cross-sectional study [33-35]. We suggest that kidney dysfunction can be prevented by interventions such as medication or lifestyle modification in hypertensive patients to maintain $\mathrm{WBPT} \leq 50.0 \mathrm{~s}$.

The present study has several limitations. First, the drug treatments for hypertension, diabetes mellitus, and dyslipidemia may have influenced the study results. Second, angiography, computed tomography, and magnetic resonance imaging were not performed. Therefore, asymptomatic cardiovascular diseases may have gone undetected. Third, hemorheology estimated using MC-FAN is an in vitro measurement that uses artificial blood vessels; therefore, the obtained hemorheological data may differ from those obtained in vivo due to the influence of vascular factors, such as endothelial cells or smooth muscle cells. However, the results of the present study indicate that WBPT is an important marker which is associated with 
kidney function. Finally, the present study was cross-sectional and was conducted at a single unit with a small sample volume. Examination of a large number of prospective studies including intervention therapies will be required to clarify the relationship between WBPT and kidney function in hypertensive patients.

\section{Conclusions}

In conclusion, the results of the present study reveal that WBPT estimated by the microchannel method is an important predictor of albuminuria or increased renovascular resistance, suggesting that WBPT may have clinical utility in preventing progression of kidney dysfunction in hypertensive patients.

\section{Conflicts of Interest}

Author has no competing interests.

\section{Grant Support}

None.

\section{Financial Disclosure}

None.

\section{References}

1. Bouchi R, Babazono T, Yoshida N, Nyumura I, Toya K, Hayashi T, Hanai K, et al. Association of albuminuria and reduced estimated glomerular filtration rate with incident stroke and coronary artery disease in patients with type 2 diabetes. Hypertens Res. 2010;33(12):1298-1304.

2. Viazzi F, Leoncini G, Derchi LE, Pontremoli R. Ultrasound Doppler renal resistive index: a useful tool for the management of the hypertensive patient. J Hypertens. 2014;32(1):149-153.

3. Cowan AQ, Cho DJ, Rosenson RS. Importance of blood rheology in the pathophysiology of atherothrombosis. Cardiovasc Drugs Ther. 2012;26(4):339-348.

4. Tzoulaki I, Murray GD, Lee AJ, Rumley A, Lowe GD, Fowkes FG. Relative value of inflammatory, hemostatic, and rheological factors for incident myocardial infarction and stroke: the Edinburgh Artery Study. Circulation. 2007;115(16):2119-2127.

5. Kikuchi Y, Sato K, Mizuguchi Y. Modified cell-flow microchannels in a single-crystal silicon substrate and flow behavior of blood cells. Microvasc Res. 1994;47(1):126139.

6. Satoh N, Kotani K, Wada H, Himeno A, Shimada S, Sasaki Y, Yamada K, et al. Unfavorable blood rheology is closely associated with arterial stiffness in obese patients.
Endocr J. 2009;56(7):915-918.

7. Hitsumoto T. Factors affecting impairment of blood rheology in obese subjects. J Cardiol. 2012;60(5):401-406.

8. Matsuo K, Ueda Y, Nishio M, Hirata A, Asai M, Nemoto $\mathrm{T}$, Kashiwase K, et al. Thrombogenic potential of whole blood is higher in patients with acute coronary syndrome than in patients with stable coronary diseases. Thromb Res. 2011;128(3):268-273.

9. Hitsumoto T. Blood rheology in female with hypercholesterolemia. Anti-Aging Med. 2012;10(2):10-15.

10. Imai $\mathrm{E}$, Horio $\mathrm{M}$, Nitta $\mathrm{K}$, Yamagata $\mathrm{K}$, Iseki $\mathrm{K}$, Hara $\mathrm{S}$, Ura N, et al. Estimation of glomerular filtration rate by the MDRD study equation modified for Japanese patients with chronic kidney disease. Clin Exp Nephrol. 2007;11(1):41-50.

11. Tublin ME, Bude RO, Platt JF. Review. The resistive index in renal Doppler sonography: where do we stand? AJR Am J Roentgenol. 2003;180(4):885-892.

12. Meerwaldt R, Links TP, Graaff R, Hoogenberg $K$, Lefrandt JD, Baynes JW, Gans RO, et al. Increased accumulation of skin advanced glycation end-products precedes and correlates with clinical manifestation of diabetic neuropathy. Diabetologia. 2005;48(8):1637-1644.

13. Matthews DR, Hosker JP, Rudenski AS, Naylor BA, Treacher DF, Turner RC. Homeostasis model assessment: insulin resistance and beta-cell function from fasting plasma glucose and insulin concentrations in man. Diabetologia. 1985;28(7):412-419.

14. Friedewald WT, Levy RI, Fredrickson DS. Estimation of the concentration of low-density lipoprotein cholesterol in plasma, without use of the preparative ultracentrifuge. Clin Chem. 1972;18(6):499-502.

15. Cesarone MR, Belcaro G, Carratelli M, Cornelli U, De Sanctis MT, Incandela L, Barsotti A, et al. A simple test to monitor oxidative stress. Int Angiol. 1999;18(2):127-130.

16. Yoshida K, Kimura T, Aoki T, Tsunekawa K, Araki O, Shoho Y, Nara M, et al. Fasting serum insulin levels and insulin resistance are associated with blood rheology in Japanese young adults without diabetes. J Int Med Res. 2016;44(3):496-507.

17. Ito S, Nagasawa T, Abe M, Mori T. Strain vessel hypothesis: a viewpoint for linkage of albuminuria and cerebrocardiovascular risk. Hypertens Res. 2009;32(2):115-121.

18. Pedrinelli R, Giampietro O, Carmassi F, Melillo E, Dell'Omo G, Catapano G, Matteucci E, et al. Microalbuminuria and endothelial dysfunction in essential hypertension. Lancet. 1994;344(8914):14-18.

19. Yagi H, Sumino H, Aoki T, Tsunekawa K, Araki O, Kimura T, Nara M, et al. Impaired blood rheology is associated with endothelial dysfunction in patients with coronary risk factors. Clin Hemorheol Microcirc. 2016;62(2):139150.

20. Ikee R, Kobayashi S, Hemmi N, Imakiire T, Kikuchi Y, Moriya H, Suzuki S, et al. Correlation between the resistive index by Doppler ultrasound and kidney function and histology. Am J Kidney Dis. 2005;46(4):603-609.

21. Linde T, Sandhagen B, Hagg A, Morlin C, Wikstrom B, Danielson BG. Blood viscosity and peripheral vascular resistance in patients with untreated essential hyperten- 
sion. J Hypertens. 1993;11(7):731-736.

22. Bohlender JM, Franke S, Stein G, Wolf G. Advanced glycation end products and the kidney. Am J Physiol Renal Physiol. 2005;289(4):F645-659.

23. Thomas MC. Advanced glycation end products. Contrib Nephrol. 2011;170:66-74.

24. Meerwaldt R, Graaff R, Oomen PHN, Links TP, Jager JJ, Alderson NL, Thorpe SR, et al. Simple non-invasive assessment of advanced glycation endproduct accumulation. Diabetologia. 2004;47(7):1324-1330.

25. Morigi M, Angioletti S, Imberti B, Donadelli R, Micheletti G, Figliuzzi M, Remuzzi A, et al. Leukocyte-endothelial interaction is augmented by high glucose concentrations and hyperglycemia in a NF-kB-dependent fashion. J Clin Invest. 1998;101(9):1905-1915.

26. Hasegawa Y, Suehiro A, Higasa S, Namba M, Kakishita E. Enhancing effect of advanced glycation end products on serotonin-induced platelet aggregation in patients with diabetes mellitus. Thromb Res. 2002;107(6):319-323.

27. Rubattu S, Pagliaro B, Pierelli G, Santolamazza C, Castro $\mathrm{SD}$, Mennuni S, Volpe M. Pathogenesis of target organ damage in hypertension: role of mitochondrial oxidative stress. Int J Mol Sci. 2014;16(1):823-839.

28. Sinha N, Dabla PK. Oxidative stress and antioxidants in hypertension-a current review. Curr Hypertens Rev. 2015;11(2):132-142.

29. Kucukatay V, Bor-Kucukatay M, Gundogdu G, Erken G,
Ozcan TO, Miloglu FD, Kadioglu Y. Vitamin E treatment enhances erythrocyte deformability in aged rats. Folia Biol (Praha). 2012;58(4):157-165.

30. Gyawali P, Richards RS. Association of altered hemorheology with oxidative stress and inflammation in metabolic syndrome. Redox Rep. 2015;20(3):139-144.

31. Tzoulaki I, Murray GD, Lee AJ, Rumley A, Lowe GD, Fowkes FG. Inflammatory, haemostatic, and rheological markers for incident peripheral arterial disease: Edinburgh Artery Study. Eur Heart J. 2007;28(3):354-362.

32. Podrez EA, Byzova TV, Febbraio M, Salomon RG, Ma Y, Valiyaveettil M, Poliakov E, et al. Platelet CD36 links hyperlipidemia, oxidant stress and a prothrombotic phenotype. Nat Med. 2007;13(9):1086-1095.

33. Yamada K, Hirayama T, Hasegawa Y. Antiplatelet effect of losartan and telmisartan in patients with ischemic stroke. J Stroke Cerebrovasc Dis. 2007;16(5):225-231.

34. van der Loo B, Spring S, Koppensteiner R. High-dose atorvastatin treatment in patients with peripheral arterial disease: effects on platelet aggregation, blood rheology and plasma homocysteine. Clin Hemorheol Microcirc. 2011;47(4):241-251.

35. Shimada S, Hasegawa K, Wada H, Terashima S, SatohAsahara N, Yamakage H, Kitaoka S, et al. High blood viscosity is closely associated with cigarette smoking and markedly reduced by smoking cessation. Circ J. 2011;75(1):185-189. 